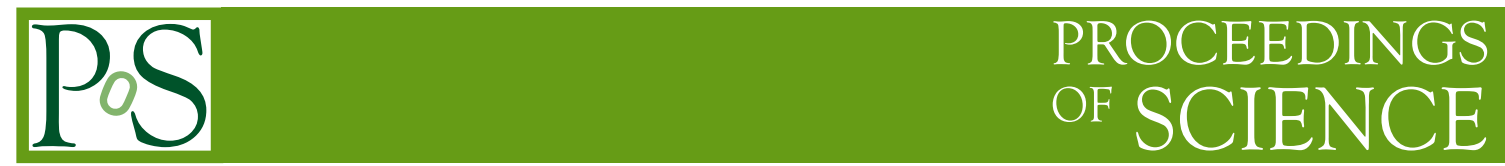

\title{
Alignment and calibration of the CMS detector
}

\author{
Gianluca Cerminara* for the CMS Collaboration \\ CERN \\ E-mail: gianluca.cerminara@cern.ch
}

Fast and efficient methods for the calibration and the alignment of the detector are a key asset to exploit the physics potential of the CMS experiment. A powerful framework, based on dedicated skims providing a highly compact dedicated input for the various calibration workflows, has been setup on this purpose. This includes a prompt calibration concept, which allows for a fast turnaround of the calibration process which is instrumental to ensure timely preparation of results for conferences and publications. The contribution reviews the design of the system and reports on the experience gained during its operation including results from selected workflows.

The 2011 Europhysics Conference on High Energy Physics-HEP 2011,

July 21-27, 2011

Grenoble, Rhône-Alpes France

\footnotetext{
*Speaker.
} 


\section{Introduction}

The design of the CMS detector [1] is based on a large super-conducting solenoid providing an intense $(3.8 \mathrm{~T})$ magnetic field, a high-precision silicon tracking system composed of about 76 millions channels including pixel and strips and hermetic calorimetry including a homogeneous Electromagnetic Calorimeter (ECAL) consisting of about $76000 \mathrm{PbWO}_{4}$ scintillating crystals. The return yoke of the solenoid houses a muon spectrometer used both for trigger and for tracking purposes.

The high level of complexity and the large number of detector channels reflect in an elaborated structure for the management and computation of the detector calibration and alignment. The present contribution reviews the workflows developed for this purpose focusing on a few selected examples.

\section{Alignment and Calibration Workflows}

Most of the alignment and calibration workflows are fed with dedicated data samples, called AlCaReco, optimized both in terms of event selection and event content. Depending on the needs of the specific workflow, these samples can be selected offline, while performing the reconstruction, or directly online, at the High Level Trigger (HLT) level. The great flexibility of the HLT, which runs offline-quality software on a farm of commercial processors, is a key asset for this online selection since it guarantees an adequate rate of events that would not be selected by the standard trigger paths meant for physics analysis.

An example of an online calibration stream is the one selecting events containing $\pi^{0}$ and $\eta$ candidates detected in ECAL and used for the inter-calibration of the $\mathrm{PbWO}_{4}$ scintillating crystals [2]. The calibration performance depends on the number of selected $\pi^{0}$ candidates per crystal and on the signal to background ratio. The candidate di-photon decays are selected at the HLT level from events passing single-e/ $\gamma$ and single-jet Level-1 triggers. After selection, only information about a limited region of ECAL (energy deposits in 20 to 40 individual crystals) near the $\pi^{0}$ candidates is stored for the actual calibration. This allows to sustain a high rate of calibration events (1 to $10 \mathrm{kHz}$ ) whilst saving bandwidth and CPU time.

\subsection{Prompt Calibration and Tier-0 processing}

Conditions changing on a short time scale require a special calibration workflow designed to allow updates with very short latency. The handling of the data streams in the first step of the offline processing on the Tier- 0 farm at CERN reflects this need and is organized as follows:

- express processing: reconstruction of a limited selection of data in order to give prompt feedback about the detector status and physics performance and to provide data for calibration workflows. The results of the express reconstruction for a given run are usually available one or two hours after the raw data are collected;

- bulk processing: reconstruction of the main data stream for physics analysis. This reconstruction step, also called prompt reconstruction, is delayed by 48 hours to allow for the computation of the fast-changing conditions. The output is divided in several Primary Datasets (PD) on the basis of the HLT paths used to select the events; 


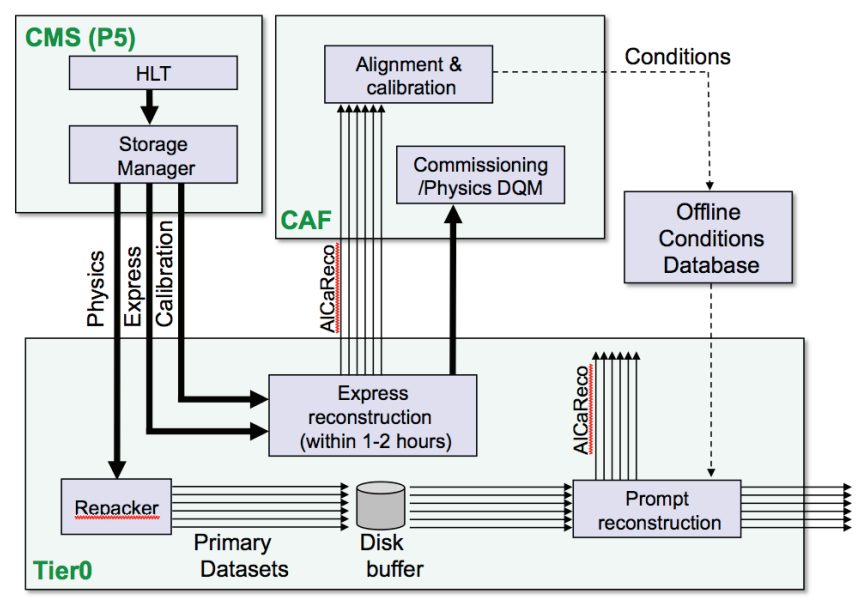

Figure 1: Data and calibration workflows during Tier-0 processing.

- calibration streams: streams of events selected at the HLT level and processed at Tier-0 for calibration purposes.

During normal operation of the CMS experiment about 300-400 $\mathrm{Hz}$ of data are processed in the bulk processing. Only a limited bandwidth, corresponding to about $10 \%$ of the bulk, is allocated for express processing in order to guarantee a fast reconstruction.

A selection of data from the express and calibration streams is used to compute the updated conditions for a given run while the bulk of the data is buffered on disk. The calibration workflows run on a dedicated farm at CERN called the CMS Analysis Facility (CAF). In this way the prompt reconstruction can profit from the updated constants, reducing the need for offline reprocessing of the data. This workflow is called the prompt calibration loop and is illustrated schematically in Fig. 1. The conditions currently updated through this kind of workflow are:

- measurement of the beam-line parameters;

- monitoring and masking of problematic channels of the silicon strip tracker to respond to HV trips or noise;

- transparency corrections of the $\mathrm{PbWO}_{4}$ crystals of the ECAL calorimeter.

Moreover, the delayed prompt reconstruction is aslo exploited to monitor possible movements of large structures of the silicon tracker, mainly due to thermal stress, and problematic channels in the electromagnetic and hadronic calorimeters allowing for quick reaction time in case of "hot" regions identified in the express reconstruction.

The measurement of the three-dimensional profile of the luminous region where the LHC beams collide at CMS [3] is an important component of the event reconstruction being used as an estimate of the primary interaction point prior to the reconstruction of the primary vertex. The position of the center of the luminous region and its width are determined using two independent methods with complementary systematic uncertainties. The first uses the distribution of the reconstructed primary vertices to map the shape of the beam line; the mean three-dimensional position is determined with a 3D likelihood fit. The second method exploits the correlation between the transverse impact parameter and the azimuthal angle of tracks when the beam line is displaced from the 


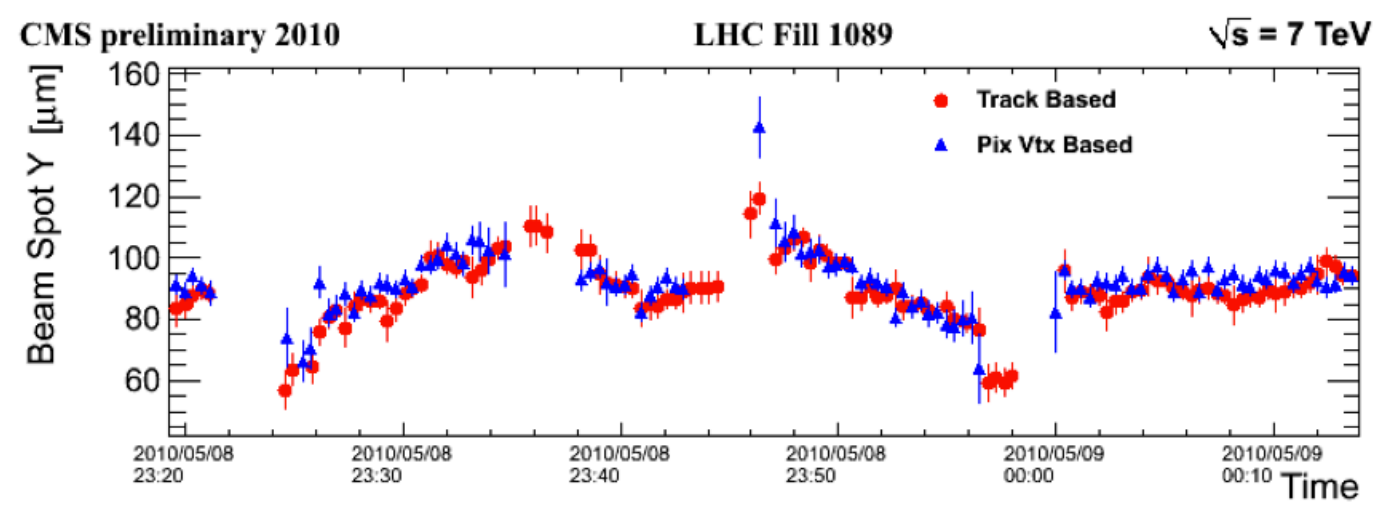

Figure 2: Fitted position of the beam line in the vertical plane as a function of time during a LHC fill where a luminosity scan was performed.

expected position. With a sample of 1000 tracks, the position can be determined with a statistical precision of about $5 \mu \mathrm{m}$.

The fit is performed once per luminosity section (corresponding to 23 seconds of data taking) using tracks selected in the express stream. In a second step, ranges with stable parameters are "collapsed", increasing the statistical precision and reducing the database storage size. Finally, the calibration object is validated and uploaded to the database. This allows the best possible knowledge of the position of the luminous region within a few hours of data being collected, with a time granularity that allows possible movements during the fill to be followed, as shown in Fig. 2.

\section{Conclusions}

The optimal alignment and calibration of each sub-detector component is a key requirement in order to achieve the full resolution and physics performance of the CMS detector. For this purpose, the collaboration set up a powerful framework that has demonstrated the required robustness and flexibility since the beginning of the LHC operation, delivering accurate calibrations with a fast turnaround and thus minimizing the need for offline reprocessing of the dataset. Nonetheless, the calibration challenge is still ongoing, and the present commitment of the CMS collaboration is to continue to optimize and consolidate all steps of the alignment and calibration workflows.

\section{References}

[1] R. Adolphi et al. [CMS Collaboration], The CMS experiment at the CERN LHC, JINST 3 (2008) S08004.

[2] The CMS Collaboration, Electromagnetic calorimeter calibration with $7 \mathrm{TeV}$ data, CMS-PAS-EGM-10-003.

[3] The CMS Collaboration, Tracking and Primary Vertex Results in First 7 TeV Collisions, CMS-PAS-TRK-10-005. 